\title{
Tropical Cyclone Rain Retrievals from $F Y-3 B$ MWRI Brightness Temperatures Using the Goddard Profiling Algorithm (GPROF)
}

\author{
RUANYU ZHANG \\ Key Laboratory of Microwave Remote Sensing, National Space Science Center, Chinese Academy of Sciences, \\ and University of Chinese Academy of Sciences, Beijing, China \\ Christian D. Kummerow, David L. Randel, Paula J. Brown, and Wesley Berg \\ Department of Atmospheric Science, Colorado State University, Fort Collins, Colorado
}

ZHENZHAN WANG

Key Laboratory of Microwave Remote Sensing, National Space Science Center, Chinese Academy of Sciences, Beijing, China

(Manuscript received 19 September 2018, in final form 26 February 2019)

\begin{abstract}
This study focuses on the tropical cyclone rainfall retrieval using $F Y-3 B$ Microwave Radiation Imager (MWRI) brightness temperatures (Tbs). The GPROF, a fully parametric approach based on the Bayesian scheme, is adapted for use by the MWRI sensor. The MWRI GPROF algorithm is an ocean-only scheme used to estimate rain rates and hydrometeor vertical profiles. An a priori database is constructed from MWRI simulated Tbs, the GPM Microwave Imager (GMI) and Dual-Frequency Precipitation Radar (DPR) combined data, and ancillary data resulting in about 100000 rainfall profiles. The performance of MWRI retrievals is consistent with DPR observations, even though MWRI retrievals slightly overestimate low rain rates and underestimate high rain rates. The total bias of MWRI retrievals is less than $13 \%$ of the mean rain rate of DPR precipitation. Statistical comparisons over GMI GPROF, GMI Hurricane GPROF (HGPROF), and MWRI GPROF retrievals show MWRI GPROF retrievals are consistent in terms of spatial distribution and rain estimates for TCs compared with the other two estimates. In terms of the global precipitation, the mean rain rates at different distances from best track locations for five TC categories are used to identify substantial differences between mean MWRI and GMI GPROF retrievals. After correcting the biases between MWRI and GMI retrievals, the performance of MWRI retrievals shows slight overestimate for light rain rates while underestimating rain rates near the eyewall for category 4 and 5 only.
\end{abstract}

\section{Introduction}

Tropical cyclones (TCs), as high-impact meteorological phenomena, occur annually in every ocean basin in the Northern Hemisphere (Gray 1968). TCs often bring great damage to coastal regions with gales, heavy precipitation, and storm surges during their landfall (Wang et al. 2007), which are responsible for a large number of casualties and vast economic losses (Zhang et al. 2009; Zick and Matyas 2016). Over the last few decades, due to increases in population and infrastructure in global coastal areas, there has been an increase in TC-related damages (Villarini et al. 2014). China is one of the

Corresponding author: Ruanyu Zhang, ruanyu_zhang@163.com world's countries suffering the most from TC disasters. Every year about 27-28 TCs form over the western North Pacific Ocean and the South China Sea, and the average number of TCs making landfall along the Chinese coast is about nine per year, according to the Yearbook of Tropical Cyclones from the China Meteorological Administration (Wang et al. 2007). Improving TCs' precipitation and track forecasts have thus become an important field of study.

Over the oceans few gauges exist, and their uneven distribution limits their usefulness for monitoring the evolution of TCs. Similarly, because of the limited range of ground-based radars, it is difficult to monitor TCs far away from land (Kidd and Levizzani 2011). Consequently, satellite instruments are being exploited 
to acquire regular global observations, especially to monitor the TCs' tracks.

The measurement of precipitation from space dates back to the advent of infrared sensors on board geostationary and low-Earth-orbiting satellites to measure cloud top temperatures (Barrett 1970). However, due to the ambiguous relationship between the information of cloud-top and surface precipitation, a large number of microwave radiometers have been developed to detect precipitation using microwave frequencies that penetrate clouds directly (Kidd et al. 2016). The TRMM Microwave Imager (TMI) and the Precipitation Radar (PR) on board the Tropical Rainfall Measuring Mission (TRMM) were used to characterize the spatial and temporal rainfall characteristics of TCs between $40^{\circ} \mathrm{N}$ and $40^{\circ} \mathrm{S}$ from 1997 to 2015 (Kummerow et al. 2001). Following the successful TRMM mission, the Global Precipitation Measurement (GPM) mission began operating in 2014. The Dual-Frequency Precipitation Radar (DPR) and GPM Microwave Imager (GMI), on board the GPM Core Observatory, provide improved capabilities for the measurement of precipitation and the microphysical properties of precipitating particles from active and passive microwave sensors (Hou et al. 2014). The $F Y-3 B$ and Fengyun-3C $(F Y-3 C)$, operating as Chinese second-generation afternoon and midmorning configured polar-orbiting satellite, were launched on 5 November 2010 and 23 September 2013, respectively. MWRIs on board both satellites are working very well and have the potential to improve precipitation estimates of TCs (Yang et al. 2012).

With the technological development of satellite instruments, precipitation retrieval algorithms have been developed and evolved to find a physical relationship between brightness temperature $(\mathrm{Tb})$ and precipitation rate. Starting with the work of Büttner (1963), work has continued over years and has led to a range of rainfall retrieval algorithms from simple emission or scattering based algorithms (Wilheit et al. 1977; Spencer et al. 1989; Kidd 1998) to multichannel physical algorithms (Tao et al. 1990; Wentz and Spencer 1998; Panegrossi et al. 1998; Olson et al. 1999; Hilburn and Wentz 2008; Kummerow et al. 2001, 2011, 2015; Hristova-Veleva et al. 2013; Kidd et al. 2016). The multichannel physically based schemes offer not only surface precipitation information but also provide vertical profile information, such as rainwater content, cloud water content, and ice water content, needed to account for the complete Tb signal. The TRMM and GPM programs use the Goddard profiling algorithm (GPROF) as the operational rainfall estimate from their passive microwave sensors.
GPROF was originally conceived by Kummerow and Giglio (1994) and devised to provide surface rain rate and latent heat release from precipitation vertical profiles. The basis of the GPROF algorithm lies in the Bayesian approach that was first adapted to precipitation retrieval by Kummerow et al. (1996). By 2014, GPROF was a fully parametric algorithm described in Kummerow et al. (2015) and successfully served a constellation of cross-track (Kidd et al. 2016) and conically scanning radiometers. The current GPROF2017 algorithm retained the GMI/DPR combined products used in the previous version (GPROF2014) but aimed to improve retrievals of high-latitude oceanic drizzle and snowfall over land (Precipitation Research Group 2017). The GPROF2017 algorithm uses an a priori database constructed from GMI/DPR combined product and hydrometeor profiles, consisting of rainwater content, cloud water content, cloud ice, and snow water content. The profiles are subset by total precipitable water (TPW), and 2-m temperature (T2m) bins based on reanalysis data, as well as 14 surface types as described by Aires et al. (2011). While the entries in a single bin sometimes exceed 300000 profiles, the profiles with similar Tbs in each bin are clustered into a maximum of 800 unique clusters. Observed Tbs are compared to Tbs in the appropriate bin and weighted by the Bayesian weight as described by Kummerow et al. (2015). The GPROF physically constrained methodology is applicable to other radiometers, as it uses radiative transfer simulations to construct databases that relate observed and simulated Tbs (Kummerow et al. 2015). This methodology was also readily adaptable to other sensors that take part in the GPM constellation, such as SSMIS (Kunkee et al. 2008), TMI (Kummerow et al. 2001, 2011), GMI (Kummerow et al. 2015), AMSR2 (Shimoda 2005), ATMS (Muth et al. 2004), and SAPHIR (Kidd et al. 2018). The addition of multiple MWRI sensors on $F Y-3 B,-3 C$, and $-3 D$ would significantly strengthen the GPM era constellation of precipitation capable sensors.

There are, unfortunately, few studies of precipitation retrievals based on MWRI observations. The literature is limited to Zhang et al. (2017) using Wentz and Spencer's algorithm (Wentz and Spencer 1998), Li et al. (2012) who retrieved TC rainfall structures from GPROF algorithm (Kummerow et al. 1996) and compared some of the MWRI rain retrievals with other rainfall products derived by AMSRE and TMI Tbs. However, the MWRI surface precipitation retrieval based on GPROF (old version) is compared with AMSRE retrieved precipitation, not radar measurements, which does not provide a systematic characterization of 
uncertainties. In addition, the GPROF algorithm was not fully parametric in that it used a simple Tb threshold or sometimes a cloud liquid water threshold (Kummerow et al. 2011) to classify pixels as raining or nonraining. These thresholds are not easily adapted to other sensors. Therefore, the purpose of this study is to incorporate the MWRI sensor into the GPROF2017 scheme in the GPM constellation era. This paper focuses on the surface precipitation retrieval of TCs over oceans by constructing an a priori database for a MWRI GPROF scheme that follows the procedures used for other imaging radiometers.

Section 2 describes the datasets used in MWRI GPROF scheme. An overview of the GPROF scheme and a new MWRI database created for TC rainfall retrieval are provided in section 3. Sections $4 \mathrm{a}$ and $4 \mathrm{~b}$ evaluate results from the new GPROF retrieval using MWRI observations. Section $4 \mathrm{c}$ presents the statistical analysis and comparisons of MWRI and GMI GPROF retrievals for TCs in five categories from 2014 to 2017. We present a discussion of the retrieval and our conclusions in section 5 .

\section{Datasets}

For the database construction, this study uses the currently available GMI GPROF2017 database for the 1-yr period (1 September 2014-31 August 2015) used by all operational GPM algorithms, but adapted to MWRI and for TCs only. The GPROF2017 database is based on the GMI and DPR combined data, the European Centre for Medium-Range Weather Forecasts (ECMWF) interim reanalysis (ERA-Interim) model data (Dee et al. 2011), and 14 surface types (Aires et al. 2011). The GPM Combined product (Precipitation Research Group 2017) consists of the surface as well as vertical hydrometer profiles. Specifically, it contains the surface precipitation plus vertical profiles of cloud and rainwater as well as a cloud ice and snow water content, all the spatial resolution of the DPR (approximately $5 \mathrm{~km} \times 5 \mathrm{~km}$ in the horizontal and $250 \mathrm{~m}$ in the vertical dimension). Together with ERA-Interim ancillary data and parameters retrieved by GMI in clear air regions, this data provides a complete description needed to compute upwelling radiances for any passive microwave sensor. This database is constructed one for each version of GPROF to incorporate the latest advances in the GPM combined algorithm and ancillary data. The adaptation is straightforward as MWRI Tbs can be computed and field of view (FOV) averaged for the available profiles. To limit the database to TCs, the best track data provided by the Joint Typhoon Warning Center (JTWC) and the National
Hurricane Center (NHC) are used to identify all pixels within $800 \mathrm{~km}$ of the TC center.

\section{a. FY-3B MWRI data}

The $F Y-3 B$ MWRI is a conically scanning passive radiometer with 10 channels (five frequencies: 10.65 , $18.7,23.8,36.5$, and $89.0 \mathrm{GHz}$, with each frequency having dual polarization). The $F Y-3 B$ MWRI has been functioning well and providing stable and continuous observations and products since its launch (November 2010). It is operating at higher stability and with lower nonlinearity compared with the MWRI onboard the $F Y-3 A$ (Yang et al. 2012). Values of the noise equivalent differential temperature (NEDT) and channel footprint of $F Y-3 B$ MWRI are provided in Table 1. The MWRI resolution is diffraction limited, with the lowest-resolution footprint $(\sim 51 \mathrm{~km} \times 85 \mathrm{~km})$ at $10.65 \mathrm{GHz}$ and the highest-resolution footprint $(\sim 9 \mathrm{~km} \times$ $15 \mathrm{~km}$ ) at the $89.0 \mathrm{GHz}$ (see Table 1). Brightness temperatures used by MWRI GPROF are given by the $F Y$-3B level-1 MWRI product, which is provided by the National Satellite Meteorological Center Feng Yun Satellite Data Center.

Because the a priori database is initially constructed using DPR and GMI observations, for the current approach it is important to ensure that MWRI's calibration is consistent with GMI. For window-channel imagers, Berg et al. (2016) developed a two-point calibration adjustment for the GPM constellation radiometers based on comparisons over oceans (cold scenes) and vegetated land surfaces (warm scenes). For $F Y-3 B$, a total of 46 months of matched observations between GMI and MWRI over the period from March 2014 through December 2017 were used to determine the intercalibration offsets in Table 2. Data over the entire GPM coverage area of $68^{\circ} \mathrm{S}$ to $68^{\circ} \mathrm{N}$ was used for the analysis. Although the $F Y-3 B \mathrm{~Tb}$ are significantly colder than GMI by as much as $5.5 \mathrm{~K}$ for the $23 \mathrm{~V}$ channel with significant scene-temperature-dependent differences, similar results were found for the AMSR2 instrument on board GCOM-W1 by the GPM intercalibration team (Berg et al. 2016). The lack of a $23.8 \mathrm{H}$ channel on GMI, however, means that this channel is not used in the creation of the a priori database nor in the precipitation retrieval. As a result, the values for the $23.8 \mathrm{H}$ calibration are "N/A" in Table 2. With the exception of the $89 \mathrm{H}$ channel, which shows a significant trend toward colder brightness temperatures for cold ocean scenes over this period, the standard deviation of the monthly intercalibration estimates between the two sensors is less than $0.5 \mathrm{~K}$. The cold-scene trend in the $89 \mathrm{H} \mathrm{Tb}$ results in a standard deviation of $1.2 \mathrm{~K}$ for that channel, however, this 
TABLE 1. Footprint size and temperature sensitivity of NEDT (K) for the MWRI channels (Yang et al. 2012).

\begin{tabular}{lcccccccccc}
\hline \multicolumn{1}{c}{ Channels } & $10.65 \mathrm{~V}$ & $10.65 \mathrm{H}$ & $18.7 \mathrm{~V}$ & $18.7 \mathrm{H}$ & $23.8 \mathrm{~V}$ & $23.8 \mathrm{H}$ & $36.5 \mathrm{~V}$ & $36.5 \mathrm{H}$ & $89.0 \mathrm{~V}$ & $89.0 \mathrm{H}$ \\
\hline NEDT $(\mathrm{K})$ & 0.30 & 0.31 & 0.35 & 0.37 & 0.36 & 0.35 & 0.26 & 0.24 & 0.55 \\
Resolution $(\mathrm{km} \times \mathrm{km})$ & $51 \times 85$ & $30 \times 50$ & $27 \times 45$ & & $18 \times 30$ & 0.64 \\
\hline
\end{tabular}

trend in the calibration is significantly less in the warmscene analysis.

\section{b. Best track data of TCs}

The latitude and longitude information of all primary TCs for the western North Pacific Ocean (WNP), the north Indian Ocean, the south Indian Ocean, and the South Pacific basins are taken from the JTWC. The JTWC maintains an archive of TCs' track data, commonly referred to as "best tracks" (Chu et al. 2002). Each best track file contains a storm best track location and intensity that are defined by a postseason analysis including surface observations, satellite images, radar images, and aircraft reports at 6-h intervals (Marino 2012). Due to the consistency of 1-min wind averaging times for all basins, JTWC data were utilized instead of the Regional Specialized Meteorological Center data (Hu et al. 2018). In addition to using TC track information to construct the a priori database, it is also used to identify TC with coincident overpasses for MWRI and GPM.

Hurricane Database 2 (HURDAT2) maintained by the NHC, a subjectively smoothed assessment of the TC best track information, provides TCs best track data for the North Atlantic and the northeast Pacific basins (Landsea and Franklin 2013). It contains the best track position, minimum pressure, maximum sustained wind, and wind parameter for storms (Klotzbach and Oliver 2015). To maximize the total number of TCs, TC best track datasets provided by both JTWC and HURDAT2 from 2014 to 2017 are used to select the number of TCs coincident with MWRI and GMI observations.

\section{MWRI GPROF scheme and database}

This study uses FY3B MWRI measurements and the GPROF2017 V1 (hereafter GPROF) to estimate TC precipitation over oceans. Kummerow et al. (2011, 2015) describe the GPROF algorithm in more detail. A general introduction of the GPROF algorithm is provided here only for completeness. Compared with the GPROF algorithm, the MWRI GPROF has been adapted to focus on surface rain rate and hydrometeor profiles from TCs and their immediate surroundings.

\section{a. The GMI GPROF database}

As an operational passive microwave rain retrieval for the GPM mission (Hou et al. 2014), the GPROF algorithm is a fully parameterized version that used the Bayesian approach for both land and ocean surfaces. As can be seen in the literatures (Kummerow et al. 2011, 2015; Kidd et al. 2016), the GPROF scheme comprises three main processing steps: the first step involves the generation of an a priori database that contains potential hydrometeor profiles along with surface rainfall rates established by the radar/radiometer combined data with appropriately simulated Tbs; the second step preprocesses the satellite measurements by attaching ancillary data (surface types and model-derived reanalysis data) to a set of Tbs for each satellite pixel; the final stage performs precipitation retrievals based on the Bayesian approach by matching the satellite observations in preprocess and the radiances in profile database with the same 2-m temperature (T2m), total precipitation water (TPW), and surface type.

The GPROF contains the GMI/DPR combined product to build a priori databases for sensors. Over oceans, hydrometeor profiles of the combined matched swath (MS) product from the DPR combined algorithm (Grecu et al. 2016) and surface precipitation derived from the combined normal swath (NS) product are used to construct a 1-yr a priori database for GMI GPROF from September 2014 to August 2015. This captures the annual cycle of precipitation in the majority of meteorological conditions. Once the radiative properties of the surface and atmosphere are known or assumed, it is straightforward to apply a radiative transfer model to

TABLE 2. $F Y-3 B$ MWRI window channel intercalibration offsets vs GMI. The final calibration offset is interpolated from the cold and warm scene values provided based on the MWRI scene temperature. Below the cold scene $\mathrm{Tb}$ and above the warm scene $\mathrm{Tb}$ the corresponding offset is applied with no extrapolation.

\begin{tabular}{lccccc}
\hline & \multicolumn{2}{c}{ Cold scene } & & \multicolumn{2}{c}{ Warm scene } \\
\cline { 2 - 3 } \cline { 6 - 6 } Channels & Offset $(\mathrm{K})$ & $\mathrm{Tb}(\mathrm{K})$ & & Offset $(\mathrm{K})$ & $\mathrm{Tb}(\mathrm{K})$ \\
\hline $10.65 \mathrm{~V}$ & 4.32 & 168.0 & & 2.94 & 279.0 \\
$10.65 \mathrm{H}$ & 5.47 & 91.0 & & 5.0 & 276.0 \\
$18.7 \mathrm{~V}$ & 0.90 & 192.0 & & 0.23 & 280.0 \\
$18.7 \mathrm{H}$ & 2.56 & 125.0 & & 2.16 & 279.0 \\
$23.8 \mathrm{~V}$ & 1.54 & 221.0 & & 1.44 & 281.0 \\
$23.8 \mathrm{H}$ & $\mathrm{N} / \mathrm{A}$ & $\mathrm{N} / \mathrm{A}$ & & $\mathrm{N} / \mathrm{A}$ & $\mathrm{N} / \mathrm{A}$ \\
$36.5 \mathrm{~V}$ & 4.64 & 214.0 & & 3.54 & 280.0 \\
$36.5 \mathrm{H}$ & 0.34 & 154.0 & & 3.80 & 278.0 \\
$89.0 \mathrm{~V}$ & 1.14 & 262.0 & & 1.40 & 282.0 \\
$89.0 \mathrm{H}$ & 2.79 & 231.0 & & 1.94 & 281.0 \\
\hline
\end{tabular}


compute the Tbs emerging at the top of the atmosphere (Kummerow et al. 1996). The one-dimensional slantpath Eddington approximation radiative transfer model is currently being used in the current GPROF to compute simulated Tbs (Kummerow et al. 1996, 2001, 2011). The detailed description of the Eddington approximation model is summarized by Kummerow (1993). Simulated Tbs and associated hydrometeor profiles are coupled with the combined NS precipitation and ancillary data to build an a priori database.

The GPROF algorithm categorized the profiles in an a priori database by T2m $(220-320 \mathrm{~K})$, TPW $(0-78 \mathrm{~mm})$, and 14 surface types. The classification of entries in the database depends on the results of Berg et al. (2006) that show regional differences in the relationship of precipitation and satellite radiances. First, the a priori database is built by aggregating all profile entries in a given $\mathrm{T} 2 \mathrm{~m} / \mathrm{TPW}$ bin to improve computational efficiency. Bin sizes of $1 \mathrm{~K}$ in T2m and $1 \mathrm{~mm}$ in TPW are used. Higher rain rate profiles contain higher TPW values (Kidd et al. 2016). However, entries in one single bin (e.g., T2m $298 \mathrm{~K}$ and TPW $45 \mathrm{~mm}$ ) sometimes exceed 300000 profiles, which are still computationally expensive (Kummerow et al. 2015). Self-similar Tbs are therefore clustered into a maximum of 800 unique clusters. The final database consists of 14 files, each one for one surface type.

\section{b. Constructing MWRI GPROF database}

Based on the GMI GPROF database, a database, consisting of precipitation and hydrometeor profiles constructed from the GMI/DPR combined product within TCs, should improve rainfall estimates from the GPROF scheme. Constructing an a priori database from a long period of DPR observations creates too many profiles, however, and is computationally very expensive. The database size used in the Bayesian retrieval scheme needs always to find a compromise between computational efficiency and representativeness (Viltard et al. 2006). Therefore, creating a new database that includes only profile entries for TCs should improve the Bayesian rainfall retrievals compared with TCs' systems, without increasing the size of the a priori database unnecessarily. Figure 1 shows the flow diagram of the MWRI database creation.

Using the GMI/DPR combined surface rain rates and hydrometer, transfer computations are carried out at the $5 \mathrm{~km}$ resolution provided by the GPM product. We simulate MWRI Tbs for the different frequency sets. MWRI Tbs are constructed by averaging each $\mathrm{Tb}$ to the corresponding channel spatial resolution, while MWRI rainfall is assumed to have the same resolution as the 18.7 GHz channel.

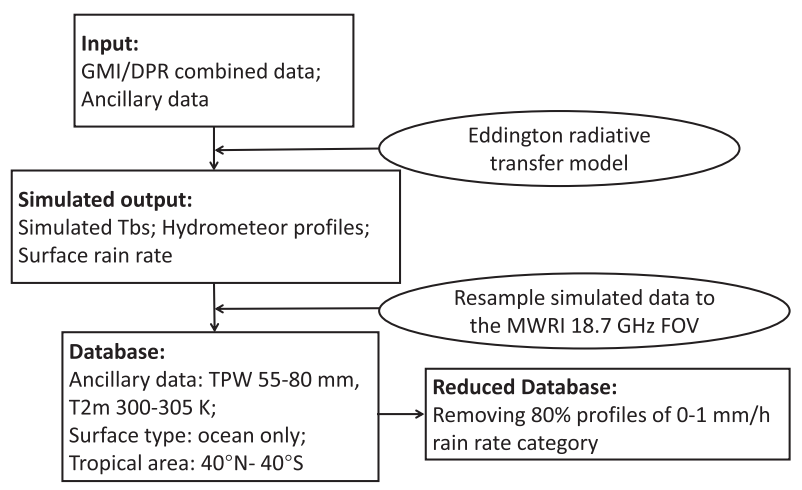

FIG. 1. Flow diagram of the MWRI database creation.

To maximize computational efficiency, the database is limited to background conditions that favor tropical cyclone development. Palmén (1948) noted that the formation and growth of TCs required the surface temperature above $26.5^{\circ} \mathrm{C}(300 \mathrm{~K})$ on tropical oceans, while Defforge and Merlis (2017) noted the TCs' genesis is not observed at the surface temperature over $32^{\circ} \mathrm{C}$ (305 K). Farfán and Fogel (2007) that TCs associated with TPW environments above $40 \mathrm{~mm}$ result in moderate or heavy precipitation events. The GMI/DPR entries with $\mathrm{T} 2 \mathrm{~m} 26.5^{\circ}-32^{\circ}(300-305 \mathrm{~K})$ and the TPW $55-80 \mathrm{~mm}$ that favor TC development and maintenance between $40^{\circ} \mathrm{S}$ and $40^{\circ} \mathrm{N}$ were therefore used in the MWRI database. It is, however, quite general and thus applicable to any precipitation under the prescribed $\mathrm{T} 2 \mathrm{~m}$ and TPW conditions. The focus on TC was intended primarily to limit the validation to specific phenomena that are fairly well observed and where properties, such as the spatial resolution of the eye and the spiral rainbands can be qualitatively compared with other sensors. Since the MWRI GPROF database contains much fewer profile entries than the GMI GPROF database, it is unnecessary to perform bin classification and clustering routines in the MWRI GPROF scheme. There are approximately 350000 profiles used in the period from September 2014 to August 2015 for the global tropics. Although the profile entries in the MWRI database are much smaller, the rain rate between 0 and $1 \mathrm{~mm} \mathrm{~h}^{-1}$ represents about $86.5 \%$ of the total entries, which is still computationally expensive. Because the very low rain rates $\left(0-1 \mathrm{~mm} \mathrm{~h}^{-1}\right)$ have similar Tbs, randomly removing $80 \%$ of the lowest rain rate $\left(0-1 \mathrm{~mm} \mathrm{~h}^{-1}\right)$ profile entries in the database increases computational efficiency and causes little deviation to TC precipitation retrievals. The remaining entries, of course, are counted by the proper ration so as not to change a priori rain rate statistics. The remaining profiles in the $0-1 \mathrm{~mm} \mathrm{~h}^{-1}$ category are assigned a weight of 5 to ensure that the rainfall statistics are not skewed by the removal of $80 \%$ of the low rain entries. 
This procedure causes little deviation to TC precipitation retrievals while speeding the retrieval up by nearly a factor of 4 .

A surface rainfall histogram provided by the MWRI GPROF database is displayed in Fig. 2. The average rain rate of all rain rate entries is $1.64 \mathrm{~mm} \mathrm{~h}^{-1}$ and the maximum rain rate is $64.33 \mathrm{~mm} \mathrm{~h}^{-1}$ in the database. Despite the large number (approximately 100000 ) of entries in the database, the distribution of light rain rates is still highly skewed with relatively few measurements at the high precipitation distribution. The distribution shape of histogram does not differ when additional profiles are included (Viltard et al. 2006). The approximately 100000 profiles in the database are therefore considered representative and adequate.

\section{c. The MWRI retrieval}

The MWRI GPROF retrieval is based on Bayesian scheme, and the detailed description of resultant retrieval algorithm is described in Kummerow et al. (1996, 2001). The function of Bayesian weight $w$ is defined as

$$
w_{i}=\exp \left[\sum_{l=1}^{10}-0.5 \frac{1}{\sigma_{l}^{2}}\left(\mathrm{~Tb}_{l, \mathrm{obs}}-\mathrm{Tb}_{l, i}\right)^{2}\right] .
$$

In Eq. (1), $i$ is an entry of the a priori database, $l$ represents radiometer channels, $\sigma_{l}$ is the channel uncertainty (temperature sensitivity of NEDT) for channel $l$, and the forward model errors estimated from the difference between observed and simulated Tbs. Parameters $\mathrm{Tb}_{l, \mathrm{obs}}$ and $\mathrm{Tb}_{l, i}$ are the observed $\mathrm{Tb}$ and the database $\mathrm{Tb}$, respectively. In the retrieval, retrieved rain rate $R$ [see Eq. (2)] is a weighted average of the entire spectrum of precipitation values in the database where each value is assigned a weight $w_{i}$,

$$
R=\frac{\sum_{i \in \mathrm{Dbase}} R_{i} w_{i}}{\sum_{i \in \mathrm{Dbase}} w_{i}}
$$

where parameter $R_{i}$ is the rain rate of the $i$ th entry in the database. While the Bayesian method generally offers excellent rain estimates, the rain rate is always a weighted mean value of the a priori statistics and mainly defined by the most frequently observed rain rates. Precipitation, underrepresented or nonexistent in the a priori database, will thus be assigned a low or even zero probability because of the low a priori probability of the observed $\mathrm{Tb}$. This is sometimes problematic when retrievals of TCs are sought, as their higher rain rates could be underrepresented in a global database. Since rainfall retrieval biases are directly derived from the Bayesian approach, they can be reduced, but not

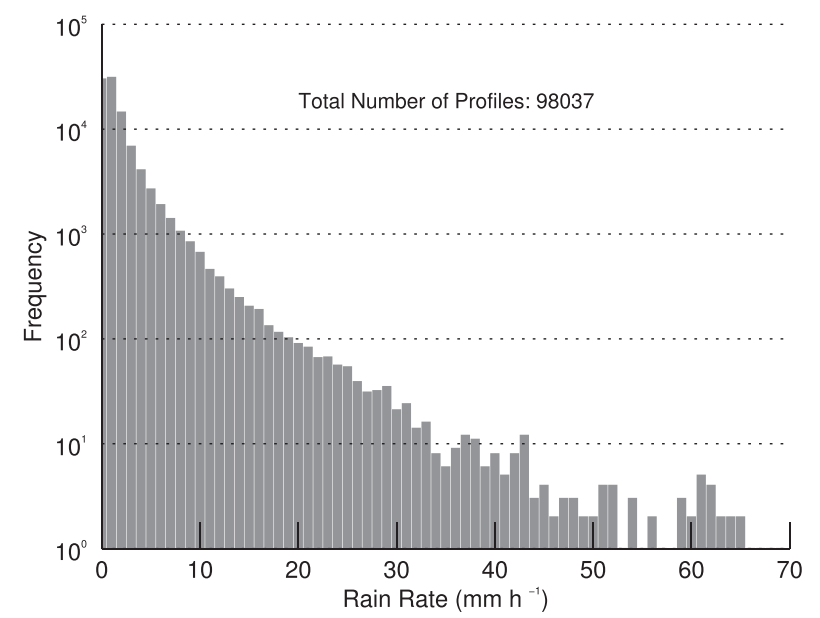

FIG. 2. Histogram of rain rates in the MWRI database.

eliminated (Petković et al. 2018). The performance of Bayesian retrieval will be significantly improved if an a priori database has richer profile entries of rain information.

The MWRI GPROF scheme currently relies on the database derived from the GPROF algorithm as described above. The JTWC and NHC best track data are used to limit retrievals to TCs. The time offset of the best track data and the scan time of MWRI coincident overpasses is always within $3 \mathrm{~h}$. MWRI observed pixels within a radius of $700 \mathrm{~km}$ (half of MWRI's swath of $1400 \mathrm{~km}$ ) of the TC's best track location are selected. Then, the MWRI observations for TCs are read into the MWRI GPROF scheme, and surface precipitation rates are retrieved based on the a priori database for TCs. Minor modifications are made for the GPROF algorithm to utilize the MWRI database. In particular, the changes affect how channel uncertainties are incorporated into retrievals. The NEDT associated with the measurement errors of the 10 MWRI's channels are listed in Table 1. The MWRI database will be applied to TCs in the WNP as examples to assess the performance of TC surface precipitation retrievals. The a priori database will also be applied to the rain rate estimates of global TCs observed by MWRI instrument from 2014 to 2017, which are compared with surface precipitation rates retrieved by GMI.

\section{Validation}

Validation of the MWRI GPROF includes three separate tasks. The first is to compare the MWRI GPROF rain retrievals to the DPR which is averaged to MWRI FOV (hereafter, DPRavgFOV). This is done for eight selected TCs. For the validation, the GMI GPROF 
(Kummerow et al. 2015) and Hurricane GPROF, HGPROF (Brown et al. 2016) precipitation retrievals are used. The second activity compares MWRI GPROF rain statistics directly to GMI GPROF and HGPROF. The last part compares rainfall histograms of global TCs between MWRI and GMI observations over the 4-yr period from 2014 to 2017, respectively, to see the deviations between these two retrievals.

\section{a. Precipitation retrieval compared with $D P R$}

The algorithm is applied to eight TC cases to illustrate the surface rain rate and structure of the resulting rain field. Before utilizing the TC identification method described in section $3 \mathrm{c}$, the TCs with coincident $(\Delta t<1 \mathrm{~h})$ observations by MWRI and GMI were identified. The eight selected TCs identified from the JTWC best track data coincident with MWRI and GPM observations are chosen as representative case study (see Table 3 ). These cases offer both a series of well-defined characteristic structures and a large range of rain intensities to verify the quality of MWRI GPROF. Since the DPR swath width is only $245 \mathrm{~km}$ (13.6 GHz, DPR Ku band), which is much smaller than that of MWRI $(\sim 1400 \mathrm{~km})$, the matched data of DPR and MWRI observations are obtained based on DPR observed scan points.

The DPR rain rates of the eight TCs in Fig. 3 show clear storm structures. The maximum rain rate is $142.3 \mathrm{~mm} \mathrm{~h}^{-1}$ occurred in TC Choiwan (orbit 09070), which is questionable at $5 \mathrm{~km}$ resolution, yet plausible in a super TC environment. Rain rates between 0 and $8 \mathrm{~mm} \mathrm{~h}^{-1}$ extend over a bulk of the TC system. The DPR rain rates are averaged to match the spatial resolution of MWRI GPROF. The DPRavgFOV rain rates are estimated by making average rain rates of all the radar data fully within MWRI $18.7 \mathrm{GHz}$ footprint $(50 \mathrm{~km} \times 30 \mathrm{~km})$. The DPRavgFOV's structures show that TC eyes (Bavi orbit 05873 and Goni orbit 08441) are free of precipitation and surrounded by eyewalls that include the strongest rain intensity. Compared with DPRavgFOV rain rates, MWRI retrieved rain rates reproduce the TC's main features, but show some differences in the rainbands. However, MWRI's lower spatial resolution (based on the resolution of $18.7 \mathrm{GHz}$ frequency) means that its rainfall structures are more smoothed compared with that of DPR measurements (Kidd et al. 2016). Due to the small time difference between MWRI and DPR observations (the time bias is controlled within $1 \mathrm{~h}$ in matchup process), MWRI TC precipitation structures have a slight displacement compared with DPR observations. Within the 1 -h time interval, the overall spatial distribution of TC precipitation does not undergo significant changes. Generally, the retrieval algorithm tends to underestimate the high rain rate and
TABLE 3. The eight selected TCs with coincident overpasses for MWRI and GPM. The DPR orbit No. is the orbit identifier.

\begin{tabular}{lcl}
\hline \hline Storm name & DPR orbit No. & \multicolumn{1}{c}{ Date } \\
\hline Bavi & 05873 & 12 Mar 2015 \\
Chanhom & 07734 & 9 Jul 2015 \\
Goni & 08441 & 24 Aug 2015 \\
Atsani & 08455 & 25 Aug 2015 \\
Choiwan & 09070 & 3 Oct 2015 \\
Lionrock & 14066 & 19 Aug 2016 \\
Lionrock & 14128 & 23 Aug 2016 \\
Lionrock & 14174 & 26 Aug 2016 \\
\hline
\end{tabular}

slightly overestimate the low end of the scale, which is attributable to the Bayesian approach. This can be expected as a radiometer is not as capable as rain sensor as the radar on GPM, even if is averaged to the same spatial resolution.

Figure 3 also shows the difference between MWRI retrievals and DPRavgFOV rain rates, keeping in mind the limited swath of DPR instrument. Although the highest rain rates are not reproduced, MWRI GPROF produces a reasonable representation of TC's precipitation rates and structures when compared with direct measurements of DPRavgFOV precipitation. The retrievals of these eight TC cases exhibit overestimates at low rainfall intensities. The high uncertainty associated with low precipitation is due to the sensitivity of MWRI Tbs to environmental parameters, such as sea surface temperature, cloud liquid water, temperature profiles, and water vapor (Viltard et al.2006). For the case of TC Bavi (orbit 05873), the MWRI retrieval shows underestimate at rain rates over $8 \mathrm{~mm} \mathrm{~h}^{-1}$ due to the Bayesian scheme. The underestimate of high precipitation rates is ascribed to the ambiguous relationship between entries with the same Tbs but contain very different rain rates. These rain rates in the database with the same probability are averaged based on the Bayesian approach, which results in intermediate values that do not represent true rainfall conditions. Another cause of underestimating high rain rates is attributed to the larger retrieval footprint $(50 \mathrm{~km} \times 30 \mathrm{~km}, 18.7 \mathrm{GHz})$ of MWRI than DPR $(5 \mathrm{~km} \times 5 \mathrm{~km}, 13.6 \mathrm{GHz})$.

To analyze the quality of MWRI GPROF scheme, detailed results are presented in Fig. 4 and Table 4. Figure 4 shows a scatterplot of MWRI GPROF against DPRavgFOV surface rain rates. Overestimation at the low rain rates for the eight cases is obvious, especially for the TC Goni (orbit 08441) and Choiwan (orbit 09070). For these two cases, when the DPRavgFOV precipitation accumulation is in the lowest rainfall range $\left(0.0-0.1 \mathrm{~mm} \mathrm{~h}^{-1}\right)$, the MWRI retrieval is close to nearly $1.0 \mathrm{~mm} \mathrm{~h}^{-1}$. One of the uncertainties is associated with the Bayesian scheme that always retrieves toward the 

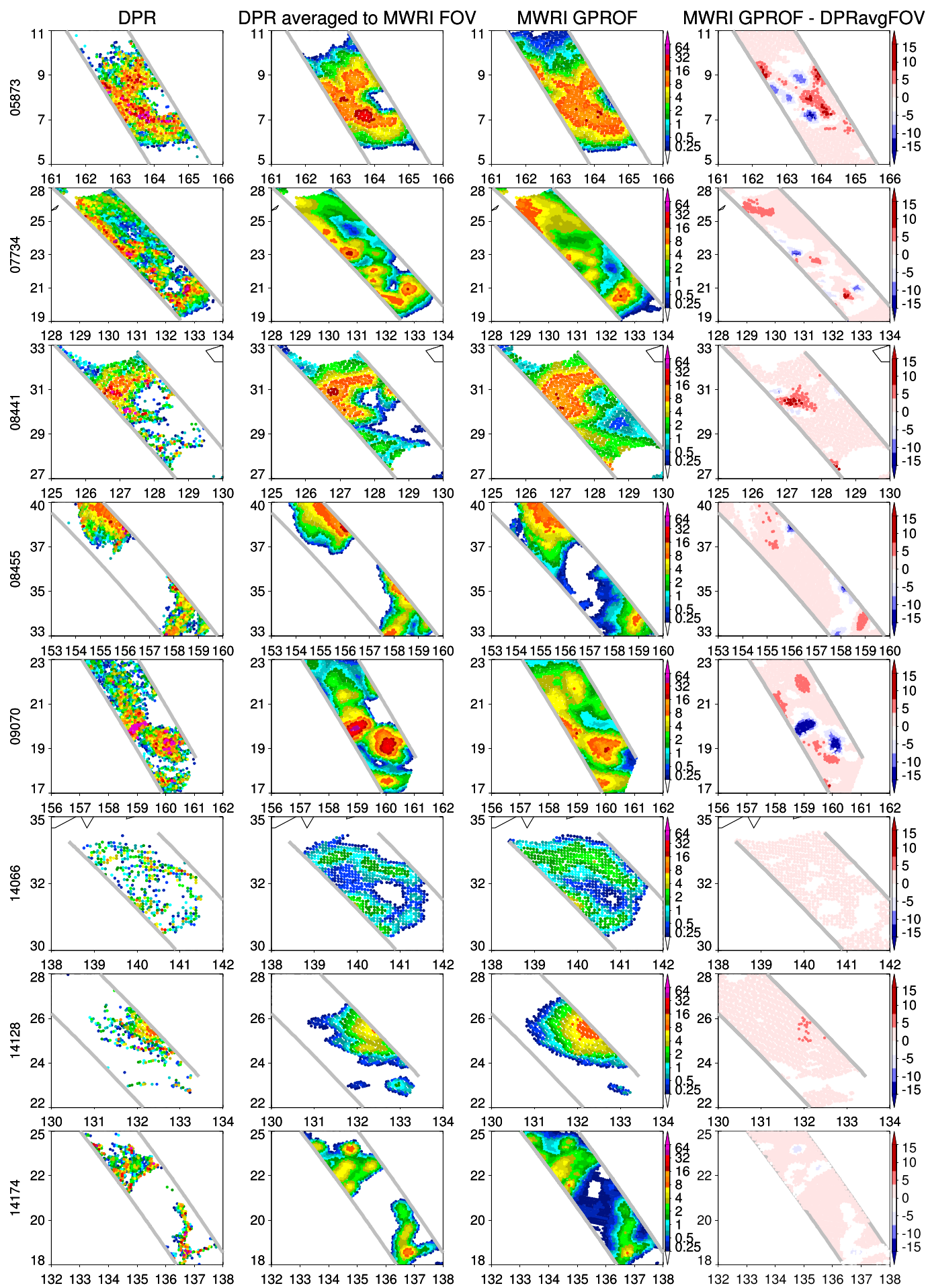

FIG. 3. Rain rates $\left(\mathrm{mm} \mathrm{h}^{-1}\right)$ for GPM DPR, DPR averaged to the MWRI FOV (DPRavgFOV), MWRI GPROF retrieval, and the differences of MWRI retrieval and DPRavgFOV for eight selected TCs.

center of rain rate distribution when less than perfect information is available. Since very lightly raining pixels have little or no brightness temperature differences from cloudy skies, the retrieval tends to exaggerate low or zero rain rate. Figure 4 also shows underestimates of high rainfall amounts except for TC Lionrock (orbit 14128). For the cases of TC Atsani (orbit 08455) and Lionrock (orbit 14174), there are obvious underestimates 

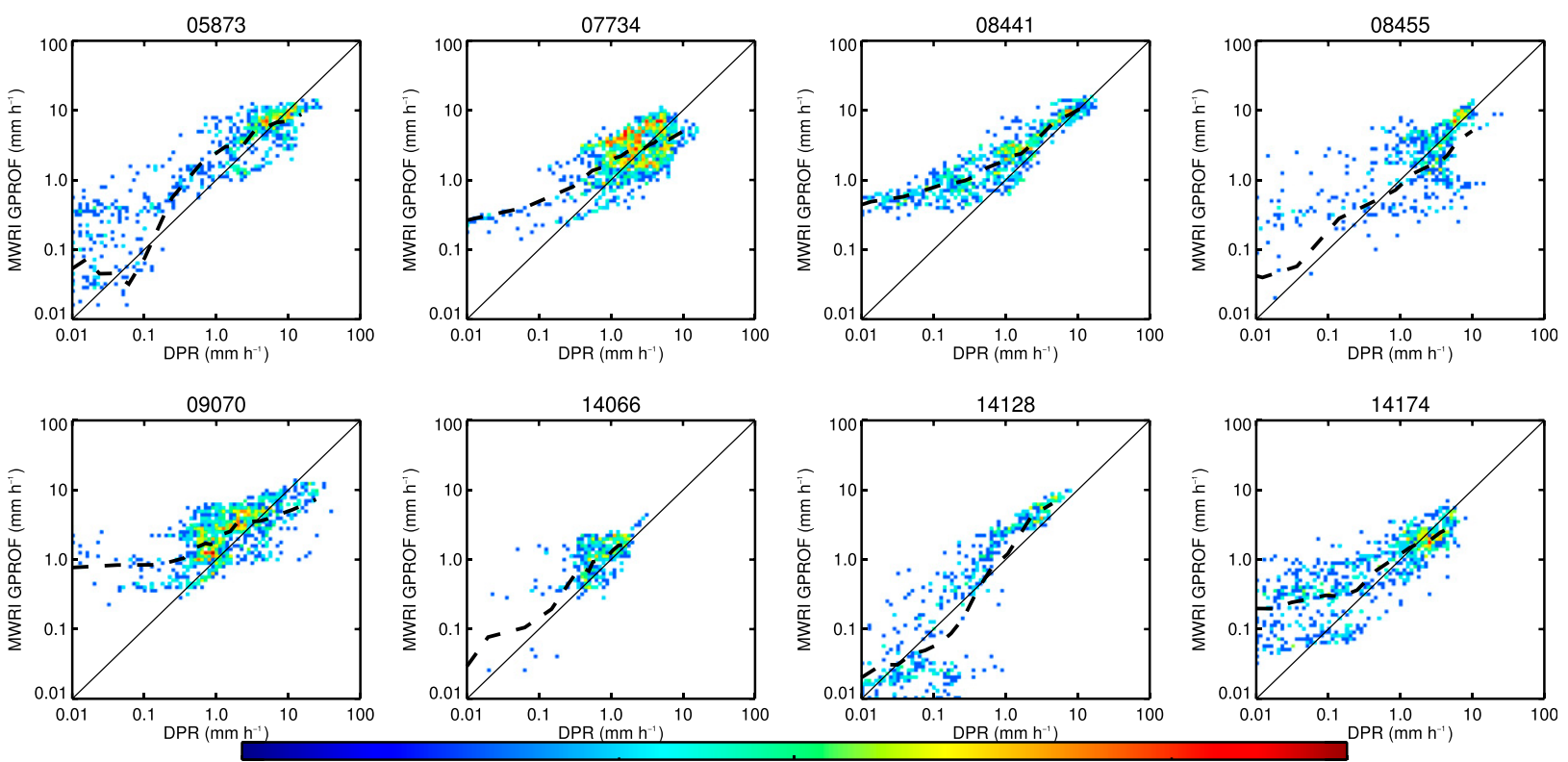

Frequency

FIG. 4. Density scatterplots of MWRI GPROF and DPRavgFOV rain rates $\left(\mathrm{mm} \mathrm{h}^{-1}\right)$ for the eight selected TCs. One-to-one line (solid line), and the mean rain rate of MWRI GPROF (dashed lines) are displayed in each TC.

compared with DPRavgFOV rain rates over $1.0 \mathrm{~mm} \mathrm{~h}^{-1}$, which is not only due lower footprint resolution of MWRI than DPR, but also the low probability of high precipitation in the database.

The statistical differences between DPRavgFOV rain rates and MWRI retrievals for each rain interval are summarized in Table 4. Five basic statistical measures are used to assess the performance of retrievals, namely, the respective mean rain rates, RMSE, MAE, and the mean bias between MWRI retrievals and DPRavgFOV rain rates. The DPRavgFOV rain rates are separated into different rainfall classifications to evaluate how MWRI GPROF works at different rain rates. The total MWRI rain rate bias shows a clear overestimation tendency (approximately 13\%). The MWRI GPROF algorithm tends to underestimate at the rain range from 5.0 to $10.0 \mathrm{~mm} \mathrm{~h}^{-1}$ and shows considerable underestimates at high rain rates above $10.0 \mathrm{~mm} \mathrm{~h}^{-1}$. The MWRI's bias and mean rain rate reveals overestimates at the rain range from 0.0 to $1.0 \mathrm{~mm} \mathrm{~h}^{-1}$ due to the Bayesian approach and information constant of the radiometer. Additionally, the relationship between a set of Tbs and rain rates in the a priori database is nonunique.

\section{b. Evaluate MWRI GPROF against GMI GPROF and $H G P R O F$}

The GPROF and HGPROF retrievals for GMI based on two different databases are used in this section. Since the GMI GPROF and HGPROF retrievals are based on two different retrieval resolutions $(10.9 \mathrm{~km} \times 18.1 \mathrm{~km}$ for $18.7 \mathrm{GHz}$ and $18.9 \mathrm{~km} \times 31.1 \mathrm{~km}$ for $21.3 \mathrm{GHz}$, respectively), these two retrievals have higher spatial resolution than the MWRI footprint $(50 \mathrm{~km} \times 30 \mathrm{~km}$, $18.7 \mathrm{GHz}$ ) and are thus averaged to the MWRI resolution before comparisons are made. Before making the comparisons of the results, the differences in the three algorithms' databases are listed in Table 5.

The precipitation rate comparisons between four products (DPRavgFOV, GMI GPROF, GMI HGPROF, and MWRI GPROF) are shown in Fig. 5 for six orbits. The results indicate that MWRI rain retrievals show good consistency with DPRavgFOV, GMI GPROF, and

TABLE 4. Quantitative assessment of MWRI GPROF retrievals compared to the DPRavgFOV rain rates $\left(\mathrm{mm} \mathrm{h}^{-1}\right)$ for eight selected TCs. The rain rate range, mean rain rates of MWRI and DPRavgFOV, mean bias (MWRI GPROF - DPRavgFOV), MAE, and RMSE are listed.

\begin{tabular}{|c|c|c|c|c|c|}
\hline \multirow[b]{2}{*}{ Rain range } & \multicolumn{2}{|c|}{ Mean rain rate } & \multirow[b]{2}{*}{ Bias } & \multirow[b]{2}{*}{ MAE } & \multirow[b]{2}{*}{ RMSE } \\
\hline & MWRI & DPRavgFOV & & & \\
\hline $0-0.1$ & 0.231 & 0.016 & 0.216 & 0.221 & 0.443 \\
\hline $0.1-0.5$ & 0.902 & 0.268 & 0.634 & 0.695 & 1.096 \\
\hline $0.5-1.0$ & 1.860 & 0.740 & 1.120 & 1.242 & 1.876 \\
\hline $1.0-5.0$ & 3.292 & 2.432 & 0.860 & 1.682 & 2.264 \\
\hline $5.0-10.0$ & 6.099 & 6.767 & -0.668 & 2.766 & 3.345 \\
\hline $10.0-64.0$ & 8.689 & 15.867 & -7.178 & 10.063 & 7.598 \\
\hline Total & 1.977 & 1.743 & 0.234 & 1.184 & 2.430 \\
\hline
\end{tabular}


TABLE 5. The differences of the MWRI GPROF, GMI GPROF, and GMI HGPROF algorithms' databases.

\begin{tabular}{lccccc}
\hline \hline \multicolumn{1}{c}{ Retrieval } & Database sensor & Database extent & Geographical extent & Footprint $(\mathrm{km} \times \mathrm{km})$ & Hydrometeor profiles \\
\hline MWRI GPORF & MWRI & 1 year & Tropics & $30 \times 50$ & DPR \\
GPROF & GMI & 1 year & Global & $10.9 \times 18.1$ & DPR \\
HGPROF & TMI & 15 years & Tropical storms & $18.9 \times 31.1$ & PR \\
\hline
\end{tabular}

GMI HGPROF rainfall products in terms of spatial distributions. Although there are time differences between the measurements of GPM GMI, DPR, and MWRI, MWRI rain rates are positively correlated with TC intensities. In addition, Fig. 5 shows a clear TC system and spatial distribution in rainfall intensities for each case. TC Bavi (orbit 05873, see Fig. 5a) shows an obvious storm system, with low precipitation near the eye (around $250-300 \mathrm{~km}$ ), and strong intensities around the eyewall. For TCs with high intensities (Figs. 5a,e), the MWRI retrievals are consistent with DPRavgFOV rain rates. However, the GMI GPROF retrievals overestimate at higher precipitation rates compared with DPRavgFOV. For lower rain intensities (Figs. 5b-d), the GMI HGPROF retrieval shows the best match with DPRavgFOV rain rates, even if there are some underestimates at rain rates around $5 \mathrm{~mm} \mathrm{~h}^{-1}$. The GMI GPROF retrievals still show significant overestimation relative to MWRI, which is mainly attributed to the smallest footprint of GMI GPROF retrievals compared with other two retrievals (see Table 5, the footprint of algorithms).

To complement the retrieval results, Fig. 6 shows comparisons between these four precipitation products for $4 \mathrm{TC}$ cases. The performance of all retrieved products is relatively consistent with DPR observations. Although the GMI GPROF, GMI HGPROF, and MWRI GPROF reproduce the main features of TCs with a clear TC structure, visible eyes and a series of rainbands, the GMI HGPROF retrieval performs much better and discerns finer detail in the rainbands close to the eyewall. The GMI GPROF algorithm produces higher estimates in high rainfall regions compared with DPRavgFOV rain rates, which is also evident in Fig. 5. While the MWRI GPROF retrieval generally resembles the DPRavgFOV precipitation, it also underestimates at high rain rates.

Figure 7 shows rainfall scatterplots of GMI GPROF, GMI HGPROF, and MWRI GPROF compared to
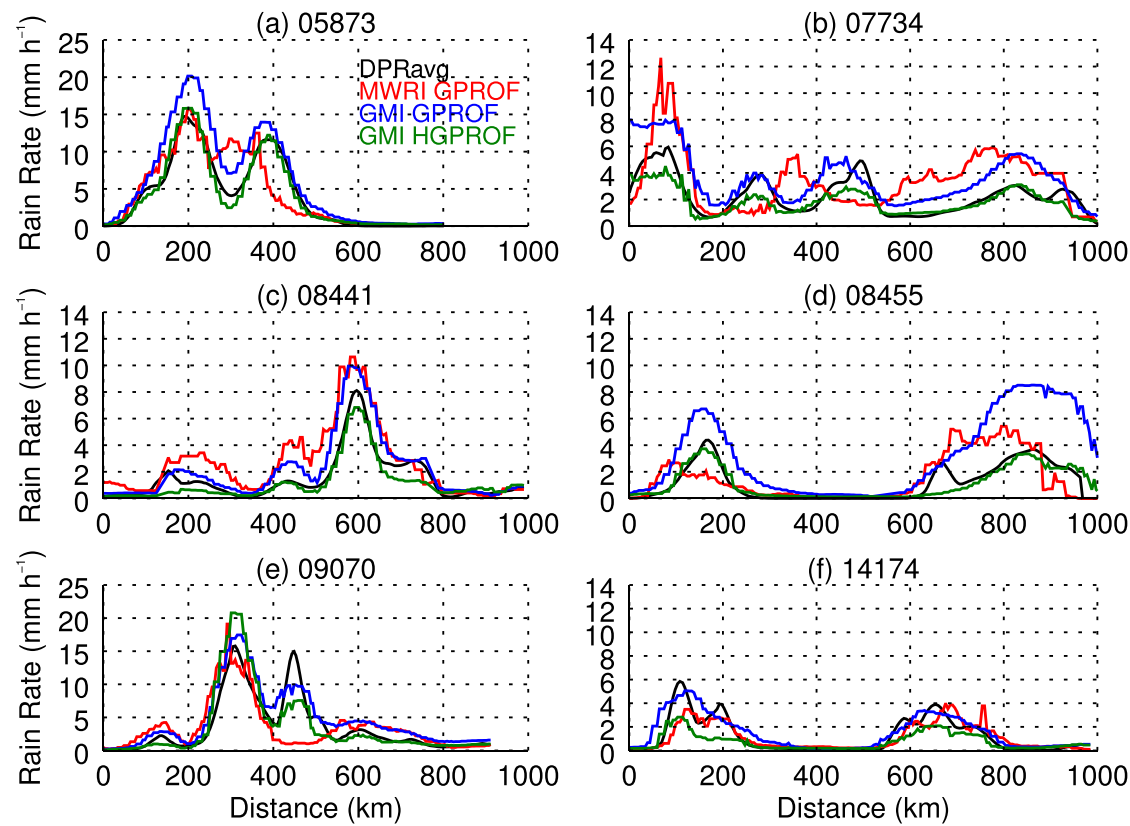

FIG. 5. Rain rates $\left(\mathrm{mm} \mathrm{h}^{-1}\right)$ assessment of DPRavgFOV, GMI GPROF, GMI HGPROF, and MWRI GPROF retrievals for six cases: (a) TC Bavi, orbit 05873; (b) TC Chanhom, orbit 07734; (c) TC Goni, orbit 08441; (d) TC Atsani, orbit 08455; (e) TC Choiwan, orbit 09070; and (f) TC Lionrock, orbit 14128. All the other three rainfall products' pixels are shown based on the center pixels (see Fig. 3) from DPRavgFOV. 

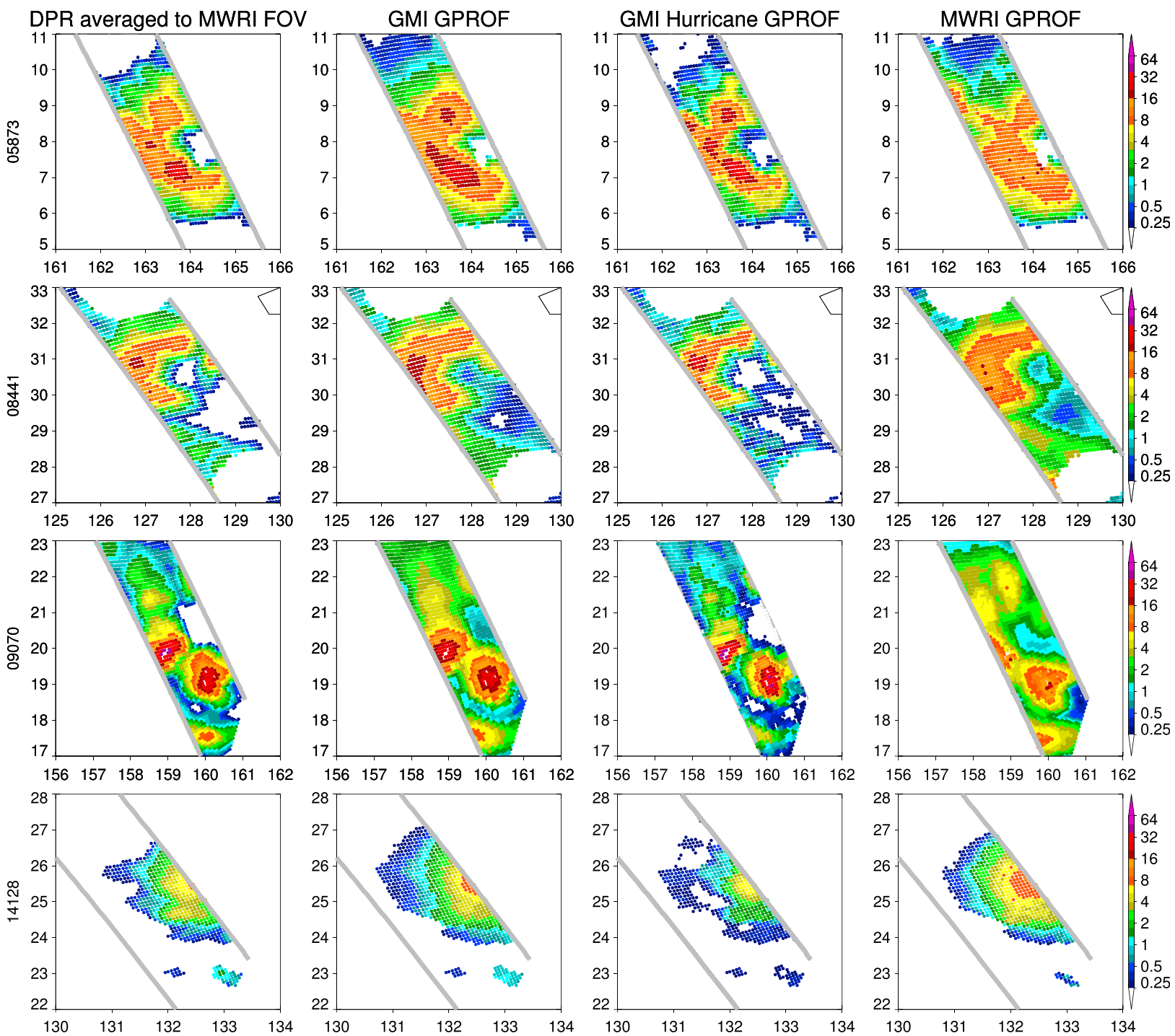

FIG. 6. Rainfall products $\left(\mathrm{mm} \mathrm{h}^{-1}\right)$ comparisons from DPRavgFOV, GMI GPROF, GMI HGPROF, and MWRI GPROF for four orbits (05873, 08441, 09070, 14128).

DPRavgFOV rain rates. GMI HGPROF is seen to produce the best fit with the DPR observations. While the GPROF retrieval matches well with the DPRavgFOV rain rate, it clearly overestimates rain rates. Even if the GMI GPROF retrieval is averaged to the MWRI's $18.7 \mathrm{GHz}$ footprint, it still exhibits higher rain rate than DPRavgFOV observed precipitation. MWRI retrievals display considerable scatter at the pixel level compared with GMI GPROF and HGPROF retrievals, which is at least in part due to the orbit time biases between MWRI and DPR measurements.

To assess the performance of these three algorithms, statistical differences are shown in Table 6. For each surface rain interval, the mean retrieved rain rate and the bias with respect to the DPR reference rain rate are presented. The low average biases of GMI HGPROF and MWRI GPROF retrievals are clearly visible. However, MWRI retrievals underestimate rain between 5 and $64 \mathrm{~mm} \mathrm{~h}^{-1}$ when compared to GMI HGPROF. The HGPROF scheme is based on a database focusing specifically on tropical storms consisting of 15 years of TRMM observations (see Table 5, database extent), containing more representative high precipitation entries than the MWRI and GMI GPROF databases that contain only 1 year of measurements. Therefore, there are not enough profile entries for high rain rates in the MWRI database. The HGPROF retrieval generally shows low MAE, RMSE, and bias compared with the other two retrievals, presenting significantly good 

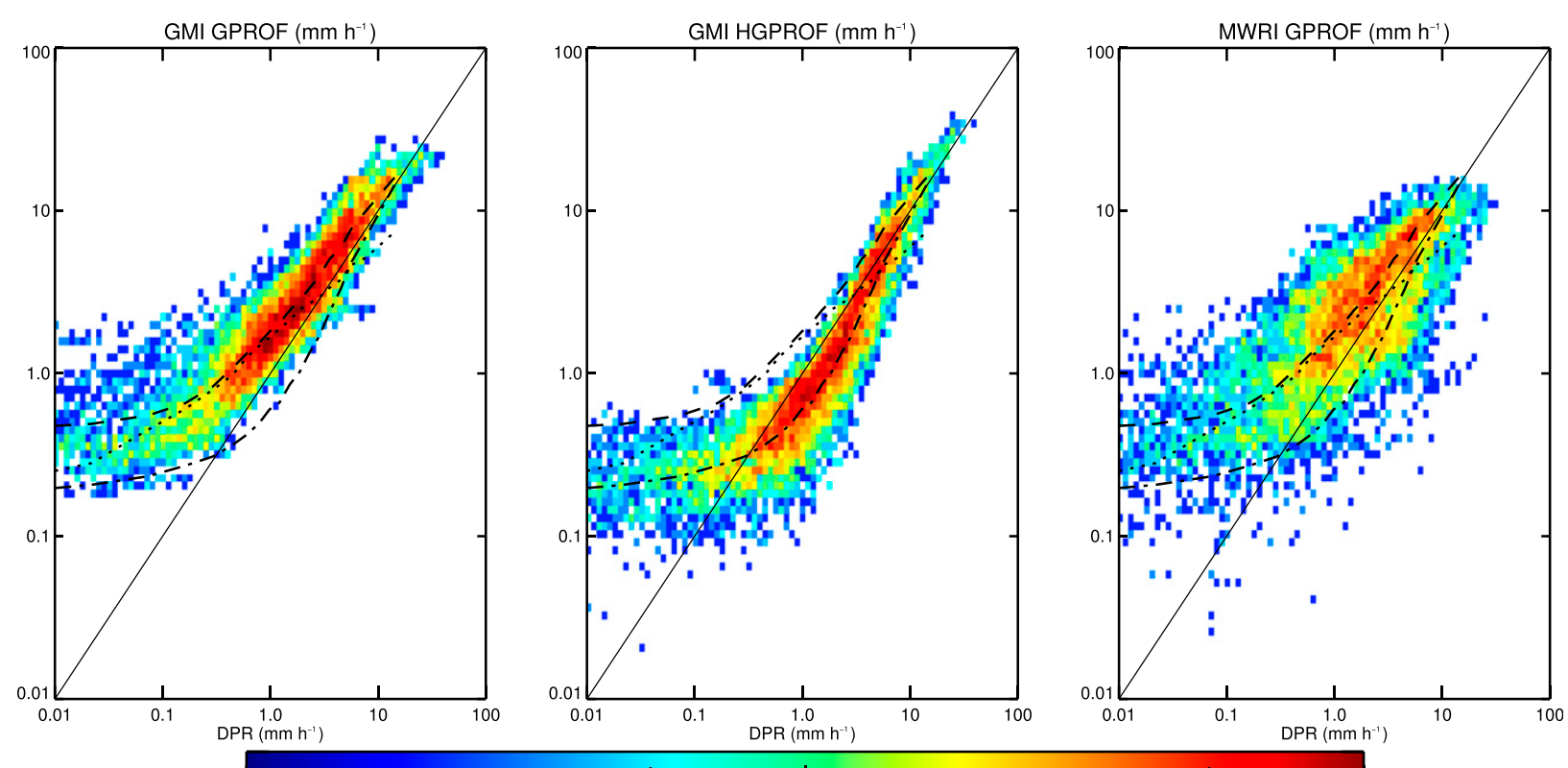

Frequency

FIG. 7. Density scatterplots of GMI GPROF, GMI HGPROF, and MWRI GPROF retrievals compared to DPRavgFOV rain rates $\left(\mathrm{mm} \mathrm{h}^{-1}\right)$ for all the eight selected TCs. One-to-one line (solid line), the mean rain rate of GMI GPROF (dashed lines), GMI HGPROF (dash-dotted lines), and MWRI GPROF (dotted lines) are displayed in each plot.

fit with the DPRavgFOV rain rate. Even if the MWRI GPROF retrieval displays higher MAE and RMSE values, it still presents good consistency with the other three products.

\section{c. Global estimation}

Despite the reasonable statistical performance of the MWRI GPROF for the eight TCs over the WNP, a key issue is whether the algorithm provides usable estimates for global TCs. In general, because the number of TCs coincident with both MWRI and GMI observations are limited, all the TCs observed by MWRI and GMI instruments from 2014 to 2017 are collected. Average rain rates in different range bins from TC's best track locations are then compared.
TC intensities are divided into five categories based on the maximum sustained wind (1-min mean) according to the Saffir-Simpson hurricane wind scale (U.S. standard). Table 7 summarizes five TC categories and the number of MWRI and GMI matched data for each category. The Saffir-Simpson hurricane wind scale used to estimate potential property damage is a 1 to 5 rating (NOAA 2005). TCs, reaching category 3 and higher, are considered to be major TCs, however, category 1 and 2 are still dangerous and require preventive measures. The TC size is classified by the radius of the area in which wind speed exceeds $15 \mathrm{~m} \mathrm{~s}^{-1}$. In this section, the radius of TCs is limited to $800 \mathrm{~km}$.

Due to the different footprints and sensor frequencies of these two retrieval schemes, there are certain

TABLE 6. Quantitative assessment of GMI GPROF, GMI HGPROF, and MWRI GPROF retrievals compared to the DPRavgFOV rain rates $\left(\mathrm{mm} \mathrm{h}^{-1}\right)$ for eight selected TCs combined. The rain rate range, mean bias, MAE, and RMSE of GMI GPROF, GMI HGPROF, MWRI GPROF, and DPRavgFOV measurements are listed.

\begin{tabular}{|c|c|c|c|c|c|c|c|c|c|}
\hline \multirow[b]{2}{*}{ Rain range } & \multicolumn{3}{|c|}{ Bias } & \multicolumn{3}{|c|}{ MAE } & \multicolumn{3}{|c|}{ RMSE } \\
\hline & GPROF & HGPROF & MWRI & GPROF & HGPROF & MWRI & GPROF & HGPROF & MWRI \\
\hline $0-0.1$ & 0.559 & 0.197 & 0.476 & 0.559 & 0.197 & 0.478 & 0.718 & 0.229 & 0.719 \\
\hline $0.1-0.5$ & 0.674 & 0.061 & 0.795 & 0.675 & 0.140 & 0.819 & 0.891 & 0.195 & 1.195 \\
\hline $0.5-1.0$ & 0.866 & -0.210 & 1.189 & 0.868 & 0.254 & 1.284 & 1.099 & 0.310 & 1.908 \\
\hline $1.0-5.0$ & 1.317 & -0.745 & 0.904 & 1.446 & 0.884 & 1.708 & 1.990 & 1.103 & 2.291 \\
\hline $5.0-64.0$ & 2.411 & -0.080 & -2.525 & 3.547 & 2.451 & 4.138 & 4.840 & 3.320 & 5.998 \\
\hline Total & 1.180 & -0.244 & 0.321 & 1.401 & 0.793 & 1.651 & 2.327 & 1.470 & 2.870 \\
\hline
\end{tabular}


TABLE 7. The categories for TCs based on sustained wind speeds. The matchup TC numbers between best track data are provided by JTWC/NHC and MWRI/GMI observations, respectively.

\begin{tabular}{|c|c|c|c|c|}
\hline \multirow[b]{2}{*}{ Category } & \multicolumn{2}{|c|}{$\begin{array}{l}\text { Sustained wind } \\
\text { speed }\end{array}$} & \multirow{2}{*}{$\begin{array}{c}\text { MWRI matched } \\
\text { TCs }\end{array}$} & \multirow{2}{*}{$\begin{array}{c}\text { GMI matched } \\
\text { TCs }\end{array}$} \\
\hline & $\mathrm{kt}$ & $\mathrm{ms}^{-1}$ & & \\
\hline 1 & $64-82$ & $33-42$ & 224 & 204 \\
\hline 2 & $83-95$ & $43-48$ & 118 & 95 \\
\hline 3 & 96-112 & $49-57$ & 92 & 61 \\
\hline 4 & $113-136$ & $58-69$ & 100 & 76 \\
\hline 5 & $>137$ & $>70$ & 21 & 17 \\
\hline
\end{tabular}

inherent differences between MWRI and GMI retrievals. For this comparison, the total rain from MWRI is scaled to match GMI. The mean precipitation rates at different distances from TCs' best track locations for each category have been calculated for the TCs coincident with MWRI and GMI observations during the period from 2014 to 2017. The comparison between MWRI and GMI retrievals for five TC categories are shown in Fig. 8. The rain rates in the central $50 \mathrm{~km}$ of each TC category show higher intensities with increasing wind speeds and the maximum mean rain rate in category 5 is nearly $25 \mathrm{~mm} \mathrm{~h}^{-1}$. Rain rates decrease with increasing distances from TCs' best track locations, and the main rain rates are concentrated in the range of $0-400 \mathrm{~km}$ from the best track location.

The comparisons between these five categories show that MWRI retrievals generally produce similar estimates with GMI rain rates, although there are some subtle differences. The performance of MWRI retrievals shows slight overestimates for the weaker TCs (generally lighter rain rates) while underestimating rain rates near the eyewall for category 4 and 5 only. The size and distribution of an a priori database have a large impact on precipitation estimates (Brown et al. 2016). As for category 4 and 5 (see Figs. 8d,e), the MWRI retrieval underestimates rain rates over $15 \mathrm{~mm} \mathrm{~h}^{-1}$ at the distance from 0 to $100 \mathrm{~km}$. The maximum rain rate of MWRI is about $9 \%$ lower than that of GMI in category 5 . The reasons for this underestimate mainly depend on three factors. First, because of the larger footprint of MWRI $(50 \mathrm{~km} \times 30 \mathrm{~km})$ than GMI $(10.9 \mathrm{~km} \times$ $18.1 \mathrm{~km})$, the GMI retrieval will estimate a higher precipitation rate. Over oceans, MWRI GPROF and GMI GPROF schemes use different combinations of channel frequencies. MWRI GPROF uses primarily channel frequencies below $89 \mathrm{GHz}$, while GMI GPROF uses frequencies not only below $89 \mathrm{GHz}$ but also $166 \mathrm{~V} / \mathrm{H}$ and $183.31 \pm 3 \mathrm{~V}, \pm 7 \mathrm{~V} \mathrm{GHz}$. The GMI observations provide more channels and each channel has a higher
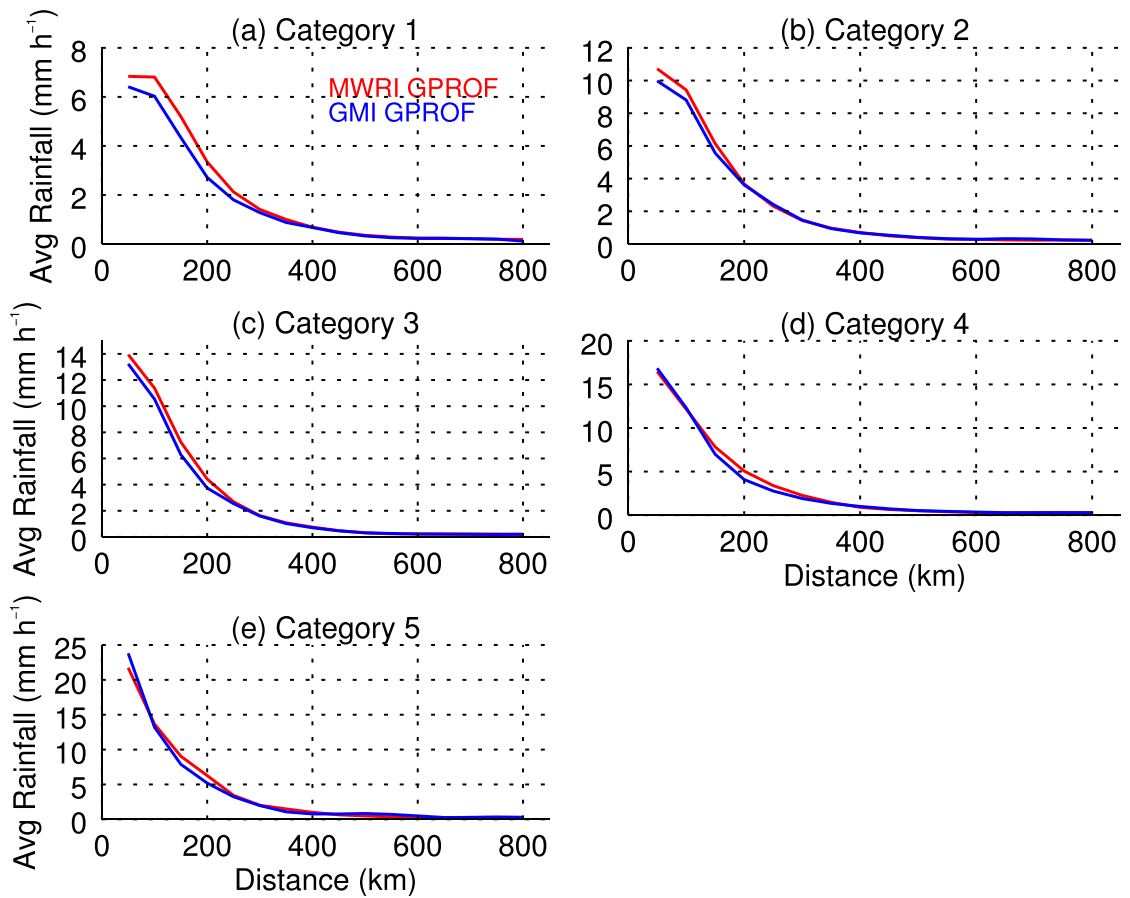

FIG. 8. Assessment of differences of the mean retrieved rain rates $\left(\mathrm{mm} \mathrm{h}^{-1}\right)$ at the different distances from the best track data for all five categories between MWRI and GMI GPROF retrievals: (a) category 1 , (b) category 2 , (c) category 3 , (d) category 4 , and (e) category 5 . The detail information of the total TC numbers coincident with MWRI and GMI sensors, respectively, during the period from January 2014 to December 2017 is related to Table 7. 
resolution than that of MWRI. Therefore, the fundamental observation-rainfall relationship will be different, and GMI GPROF retrievals have more information with which to retrieve precipitation.

\section{Discussion and conclusions}

To adapt the $F Y-3 B$ Microwave Radiation Imager (MWRI) brightness temperatures (Tbs) sensor to the current the Goddard profiling algorithm (GPROF) in the Global Precipitation Measurement (GPM) mission constellation era, this study mainly focuses on surface rainfall retrievals for tropical cyclones (TCs) using MWRI Tbs. TCs are important for a number of reasons but more frequent revisit times are a key aspect to both improved rainfall estimates and weather forecasts. The MWRI GPROF algorithm, an adaptation of the current GPROF algorithm, is an ocean-only retrieval scheme for TC precipitation and vertical hydrometeor profiles. Based on the GPROF database, an a priori database is constructed of MWRI simulated Tbs, the field of view (FOV) averaged profiles from the GPM Microwave Imager (GMI) and Dual-Frequency Precipitation Radar (DPR) combined data, and ancillary data. The generation of TCs generally occurs at total precipitation water (TPW) thresholds from 55 to $80 \mathrm{~mm}$ and $2-\mathrm{m}$ temperature $(\mathrm{T} 2 \mathrm{~m})$ thresholds from 300 to $305 \mathrm{~K}$. The a priori database is therefore limited to these conditions to constrain the total number of entries in the database. After randomly removing $80 \%$ of the profile entries of the lowest surface rain rates $\left(0-1 \mathrm{~mm} \mathrm{~h}^{-1}\right)$, a $1-\mathrm{yr}$ a priori database (September 2014 to August 2015) of MWRI GPROF is created covering part ranges of global ocean tropics $\left(40^{\circ} \mathrm{S}-40^{\circ} \mathrm{N}\right)$.

Comparisons between rain retrievals and radar observations for eight selected TCs (Fig. 3) show that MWRI GPROF retrievals are at least comparable with DPRavgFOV precipitation rates. While MWRI retrievals capture the main TC features, rain rates near the eyewall (high rain intensities) show a slight underestimation due to the low distribution of high rainfall profile entries in the database (Fig. 2). The MWRI GPROF algorithm relies on the database for physical-based rainfall retrieval within a Bayesian technique. As noted in Fig. 4 and Table 4, MWRI retrievals overestimate lower rain rates and underestimate higher rain rates of TCs. This is a common disadvantage of using a Bayesian approach to estimate rain rates at the upper and lower limits of their distribution (Brown et al. 2016). In the lowest rainfall range of $0.0-0.1 \mathrm{~mm} \mathrm{~h}^{-1}$, MWRI retrievals overestimate rainfall, which is not only due to the Bayesian retrieval scheme, but also the low sensitivity of radar datasets to very light precipitation. The underestimation of higher precipitation is caused by the saturation regime where different rain rates can represent the same Tbs. Another reason is ascribed to the larger footprint of MWRI than GMI.

Using GMI GPROF and HGPROF retrievals to assess MWRI retrieval performance, it is found that MWRI retrievals show good consistency with the other two products in terms of spatial distribution and structures for TCs (Figs. 5, 6). The GMI HGPROF retrieval outperforms other estimates at the MWRI's resolution, particularly for heavy rainband features in the inner and outer cores of TC systems. Additionally, MWRI retrievals display considerable scatter at the pixel level (Fig. 7) mainly because of the orbit time bias between MWRI and GMI/DPR sensors. Statistical comparisons (Table 6) of these three retrievals against DPRavgFOV rain rates show that the average rainfall biases of GMI HGPROF and MWRI GPROF are lower than GMI GPROF.

In terms of global precipitation, the mean rain rates at different distances from TC best track locations for all five categories of TC intensity are used to identify where substantial differences occur between MWRI and GMI GPROF retrievals. MWRI retrievals generally produce similar rainfall histograms with GMI retrieved rain rates. After correcting the overall biases between these two retrievals, the performance of MWRI GPROF shows a slight overestimation at the rainfall range of $1-15 \mathrm{~mm} \mathrm{~h}^{-1}$ for all categories (Fig. 8). On the other hand, MWRI retrievals tend to underestimate rain rates higher than $15 \mathrm{~mm} \mathrm{~h}^{-1}$ compared with GMI retrievals for categories 4 and 5, which is due to the larger footprint, less channel frequency information of MWRI than GMI, and lower distribution of high rain rates (over $15 \mathrm{~mm} \mathrm{~h}^{-1}$ ) in the database.

Acknowledgments. We thank anonymous reviewers for their constructive comments on the manuscript. This project was funded by the National Natural Science Foundation of China under Grant 61501433, and was also supported by the Chinese Scholarship Council (CSC). We thank the NASA GPM Precipitation Processing System (PPS) team and the National Satellite Meteorological Center Feng Yun Satellite Data Center for providing the satellite datasets and JTWC and NHC for the provision of best track data for TCs.

\section{REFERENCES}

Aires, F., C. Prigent, F. Bernardo, C. Jiménez, R. Saunders, and P. Brunel, 2011: A Tool to Estimate Land-Surface Emissivities at Microwave frequencies (TELSEM) for use in numerical weather prediction. Quart. J. Roy. Meteor. Soc., 137, 690-699, https://doi.org/10.1002/qj.803.

Barrett, E. C., 1970: The estimation of monthly rainfall from satellite. Mon. Wea. Rev., 98, 322-327, https://doi.org/10.1175/ 1520-0493(1970)098<0322:TEOMRF >2.3.CO;2. 
Berg, W., T. L'Ecuyer, and C. Kummerow, 2006: Rainfall climate regimes: The relationship of regional TRMM rainfall biases to the environment. J. Appl. Meteor. Climatol., 45, 434-454, https://doi.org/10.1175/JAM2331.1.

_ , and Coauthors, 2016: Intercalibration of the GPM microwave radiometer constellation. J. Atmos. Oceanic Technol., 33, 2639-2654, https://doi.org/10.1175/JTECH-D-16-0100.1.

Brown, P. J., C. D. Kummerow, and D. L. Randel, 2016: Hurricane GPROF: An optimized ocean microwave rainfall retrieval for tropical cyclones. J. Atmos. Oceanic Technol., 33, 1539-1556, https://doi.org/10.1175/JTECH-D-15-0234.1.

Büttner, K. J. K., 1963: Regenortung vom Wettersatelliten mit Hilfe von Zentimeterwellen (Rain localization from a weather satellite via centimeter waves). Naturwissenschaften, $\mathbf{5 0}$, 591-592, https://doi.org/10.1007/BF00632686.

Chu, J., C. R. Sampson, A. S. Levine, and E. Fukada, 2002: The joint typhoon warning center tropical cyclone best-tracks, 1945-2000. Doc. NRL/MR/7540-02-16, Joint Typhoon Warning Center, Naval Research Laboratory, http://www.metoc.navy.mil/jtwc/ products/best-tracks/tc-bt-report.html.

Dee, D. P., and Coauthors, 2011: The ERA-Interim reanalysis: Configuration and performance of the data assimilation system. Quart. J. Roy. Meteor. Soc., 137, 553-597, https://doi.org/ 10.1002/qj.828.

Defforge, C. L., and T. M. Merlis, 2017: Evaluating the evidence of a global sea surface temperature threshold for tropical cyclone genesis. J. Climate, 30, 9133-9145, https://doi.org/ 10.1175/JCLI-D-16-0737.1.

Farfán, L. M., and I. Fogel, 2007: Influence of tropical cyclones on humidity patterns over southern Baja California, Mexico. Mon. Wea. Rev., 135, 1208-1224, https://doi.org/10.1175/ MWR3356.1.

Gray, W. M., 1968: Global view of the origins of tropical disturbances and storms. Mon. Wea. Rev., 96, 669-700, https://doi.org/ 10.1175/1520-0493(1968)096<0669:GVOTOO > 2.0.CO;2.

Grecu, M., W. S. Olson, S. J. Munchak, S. Ringerud, L. Liao, Z. Haddad, B. L. Kelley, and S. F. McLaughlin, 2016: The GPM combined algorithm. J. Atmos. Oceanic Technol., 33, 2225-2245, https://doi.org/10.1175/JTECH-D-16-0019.1.

Hilburn, K. A., and F. J. Wentz, 2008: Intercalibrated passive microwave rain products from the Unified Microwave Ocean Retrieval Algorithm (UMORA). J. Appl. Meteor. Climatol., 47, 778-794, https://doi.org/10.1175/2007JAMC1635.1.

Hou, A. Y., and Coauthors, 2014: The global precipitation measurement mission. Bull. Amer. Meteor. Soc., 95, 701-722, https://doi.org/10.1175/BAMS-D-13-00164.1.

Hristova-Veleva, S., and Coauthors, 2013: Revealing the winds under the rain. Part I: Passive microwave rain retrievals using a new observation-based parameterization of subsatellite rain variability and intensity-Algorithm description. J. Appl. Meteor. Climatol., 52, 2828-2848, https://doi.org/ 10.1175/JAMC-D-12-0237.1.

Hu, C., C. Zhang, S. Yang, D. Chen, and S. He, 2018: Perspective on the northwestward shift of autumn tropical cyclogenesis locations over the western North Pacific from shifting ENSO. Climate Dyn., 51, 2455-2465, https://doi.org/10.1007/ s00382-017-4022-1.

Kidd, C., 1998: On rainfall retrieval using polarization-corrected temperatures. Int. J. Remote Sens., 19, 981-996, https://doi.org/ 10.1080/014311698215829.

_ trievals. Hydrol. Earth Syst. Sci., 15, 1109-1116, https://doi.org/ 10.5194/hess-15-1109-2011.
—, T. Matsui, J. Chern, K. Mohr, C. D. Kummerow, and D. L. Randel, 2016: Global precipitation estimates from cross-track passive microwave observations using a physically based retrieval scheme. J. Hydrometeor., 17, 383-400, https://doi.org/ 10.1175/JHM-D-15-0051.1.

- R. Roca, and E. Stocker, 2018: Development of the SAPHIR Precipitation Retrieval and Profiling Scheme (PRPS). Geophysical Research Abstracts, Vol. 20, Abstract EGU20189356, https://meetingorganizer.copernicus.org/EGU2018/ EGU2018-9356.pdf.

Klotzbach, P. J., and E. C. J. Oliver, 2015: Variations in global tropical cyclone activity and the Madden-Julian Oscillation since the midtwentieth century. Geophys. Res. Lett., 42, 41994207, https://doi.org/10.1002/2015GL063966.

Kummerow, C. D., 1993: On the accuracy of the Eddington approximation for radiative transfer in the microwave frequencies. J. Geophys. Res., 98, 2757-2765, https://doi.org/10.1029/ 92JD02472.

_ estimating rainfall and vertical structure information from space. Part I: Algorithm description. J. Appl. Meteor., 33, 3-18, https://doi.org/10.1175/1520-0450(1994)033<0003: APMTFE $>2.0 . \mathrm{CO} ; 2$

— W. S. Olson, and L. Giglio, 1996: A simplified scheme for obtaining precipitation and vertical hydrometeor profiles from passive microwave sensors. IEEE Trans. Geosci. Remote Sens., 34, 1213-1232, https://doi.org/10.1109/36.536538.

, and Coauthors, 2001: The evolution of the Goddard Profiling Algorithm (GPROF) for rainfall estimation from passive microwave sensors. J. Appl. Meteor., 40, 1801-1820, https://doi.org/10.1175/1520-0450(2001)040<1801:TEOTGP > 2.0.CO;2.

, S. Ringerud, J. Crook, D. L. Randel, and W. Berg, 2011: An observationally generated a priori database for microwave rainfall retrievals. J. Atmos. Oceanic Technol., 28, 113-130, https://doi.org/10.1175/2010JTECHA1468.1.

- D. L. Randel, M. Kulie, N. Wang, R. Ferraro, S. J. Munchak, and V. Petković, 2015: The evolution of the Goddard profiling algorithm to a fully parametric scheme. J. Atmos. Oceanic Technol., 32, 2265-2280, https://doi.org/ 10.1175/JTECH-D-15-0039.1.

Kunkee, D. B., G. A. Poe, D. J. Boucher, S. D. Swadley, Y. Hong, J. E. Wessel, and E. A. Uliana, 2008: Design and evaluation of the first special sensor microwave imager/sounder. IEEE Trans. Geosci. Remote Sens., 46, 863-883, https://doi.org/ 10.1109/TGRS.2008.917980.

Landsea, C. W., and J. L. Franklin, 2013: Atlantic hurricane database uncertainty and presentation of a new database format. Mon. Wea. Rev., 141, 3576-3592, https://doi.org/ 10.1175/MWR-D-12-00254.1.

Li, X., F. Zhao, Y. Qiao, H. Yang, and R. You, 2012: Comparison of three rainfall products from Microwave Imagers during development of Typhoon Ma-on. Adv. Mater. Res., 442, 167-171, https://doi.org/10.4028/www.scientific.net/ AMR.442.167.

Marino, D. R., 2012: Statistical analysis of ensemble forecasts of tropical cyclone tracks over the Northwest Pacific Ocean. Ph.D. thesis, Naval Postgraduate School, 84 pp., http://hdl.handle.net/ 10945/17412.

Muth, C., P. S. Lee, J. C. Shiue, and W. A. Webb, 2004: Advanced technology microwave sounder on NPOESS and NPP. Proc. IEEE Int. Conf. on Geoscience and Remote Sensing Symp. 2004, Anchorage, AK, Institute of Electrical 
and Electronics Engineers, 2454-2458, https://doi.org/10.1109/ IGARSS.2004.1369789.

NOAA, 2005: Saffir-Simpson hurricane wind scale. NOAA/National Hurricane Center, https://www.nhc.noaa.gov/aboutsshws.php.

Olson, W. S., C. D. Kummerow, Y. Hong, and W.-K. Tao, 1999: Atmospheric latent heating distributions in the tropics derived from satellite passive microwave radiometer measurements. J. Appl. Meteor., 38, 633-664, https://doi.org/ 10.1175/1520-0450(1999)038<0633:ALHDIT>2.0.CO;2.

Palmén, E., 1948: On the formation and structure of tropical hurricanes. Geophysica, 3 (1), 26-38.

Panegrossi, G., and Coauthors, 1998: Use of cloud model microphysics for passive microwave-based precipitation retrieval: Significance of consistency between model and measurement manifolds. J. Atmos. Sci., 55, 1644-1673, https://doi.org/ 10.1175/1520-0469(1998)055<1644:UOCMMF>2.0.CO;2.

Petković, V., C. D. Kummerow, D. L. Randel, J. R. Pierce, and J. K. Kodros, 2018: Improving the quality of heavy precipitation estimates from satellite passive microwave rainfall retrievals. J. Hydrometeor., 19, 69-85, https://doi.org/ 10.1175/JHM-D-17-0069.1.

Precipitation Research Group, 2017: NASA Global Precipitation Measurement Mission: GPROF2017. Algorithm Theoretical Basis Doc., Version 1, 63 pp., http://rain.atmos.colostate.edu/ ATBD/ATBD_GPM_June1_2017.pdf.

Shimoda, H., 2005: GCOM missions. Proc. IEEE Int. Conf. on Geoscience and Remote Sensing Symp. 2005, Seoul, South Korea, Institute of Electrical and Electronics Engineers, 4201-4204, https://doi.org/10.1109/IGARSS.2005.1525844.

Spencer, R. W., H. M. Goodman, and R. E. Hood, 1989: Precipitation retrieval over land and ocean with the SSM/I: Identification and characteristics of the scattering signal. J. Atmos. Oceanic Technol., 6, 254-273, https://doi.org/ 10.1175/1520-0426(1989)006<0254:PROLAO > 2.0.CO;2.

Tao, W.-K., J. Simpson, S. Lang, M. McCumber, R. Adler, and R. Penc, 1990: An algorithm to estimate the heating budget from vertical hydrometeor profiles. J. Appl. Meteor., 29, 1232-1244, https://doi.org/10.1175/1520-0450(1990)029<1232: AATETH $>2.0 . \mathrm{CO} ; 2$.
Villarini, G., D. A. Lavers, E. Scoccimarro, M. Zhao, M. F. Wehner, G. A. Vecchi, T. R. Knutson, and K. A. Reed, 2014: Sensitivity of tropical cyclone rainfall to idealized global-scale forcings. J. Climate, 27, 4622-4641, https://doi.org/10.1175/ JCLI-D-13-00780.1.

Viltard, N., C. Burlaud, and C. D. Kummerow, 2006: Rain retrieval from TMI brightness temperature measurements using a TRMM PR-based database. J. Appl. Meteor. Climatol., 45, 455-466, https://doi.org/10.1175/JAM2346.1.

Wang, B., Y. Xu, and B. Bi, 2007: Forecasting and warning of tropical cyclones in China. Data Sci. J., 6, S723-S737, https:// doi.org/10.2481/dsj.6.S723.

Wentz, F. J., and R. W. Spencer, 1998: SSM/I rain retrievals within a unified all-weather ocean algorithm. J. Atmos. Sci., 55, 1613-1627, https://doi.org/10.1175/1520-0469(1998)055<1613: SIRRWA $>2.0 . \mathrm{CO} ; 2$.

Wilheit, T. T., A. T. C. Chang, M. S. V. Rao, E. B. Rodgers, and J. S. Theon, 1977: A satellite technique for quantitatively mapping rainfall rates over the oceans. J. Appl. Meteor., 16, 551-560, https://doi.org/10.1175/1520-0450(1977)016<0551: ASTFQM $>2.0 . \mathrm{CO} ; 2$.

Yang, H., X. Zou, X. Li, and R. You, 2012: Environmental data records from FengYun-3B microwave radiation imager. IEEE Trans. Geosci. Remote Sens., 50, 4986-4993, https:// doi.org/10.1109/TGRS.2012.2197003.

Zhang, Q., L. Wu, and Q. Liu, 2009: Tropical cyclone damages in China 1983-2006. Bull. Amer. Meteor. Soc., 90, 489-496, https://doi.org/10.1175/2008BAMS2631.1.

Zhang, R., Z. Wang, L. Zhang, and Y. Li, 2017: Rainfall retrieval of tropical cyclones using $F Y-3 B$ microwave radiation imager (MWRI). Proc. IEEE Int. Conf. on Geoscience and Remote Sensing Symp. 2017, Fort Worth, TX, USA, Institute of Electrical and Electronics Engineers, 550-553, https://doi.org/ 10.1109/IGARSS.2017.8127012.

Zick, S. E., and C. J. Matyas, 2016: A shape metric methodology for studying the evolving geometries of synoptic-scale precipitation patterns in tropical cyclones. Ann. Amer. Assoc. Geogr., 106, 1217-1235, https://doi.org/10.1080/ 24694452.2016.1206460. 